\title{
Beyond Forms and Manuals: A Sociocognitive Approach to Academic Citation in Novice EFL Researchers' Writing*
}

\author{
Liyin Zhang \\ School of Foreign Languages \\ Northwest University \\ Xi'an, China 710127
}

\begin{abstract}
As a prominent feature of academic discourse, citation is viewed as the ability to use sources effectively and appropriately and an essential skill that novice researchers must acquire [1]. However, due to novice researchers' insufficient disciplinary and linguistic competence and their unbalanced power relations to potential readers, appropriate citation may be hard to achieve, which is particularly true for $\mathrm{L} 2$ writers. The current study focuses on novice Chinese EFL researchers, the rapidly increasing population in the world academic arena but insufficiently studied so far. We report on emic, interview-based case study investigating three novice Chinese EFL researchers' accounts of their attitudes, motives and self-initiated strategies developed upon citations in academic writing for scholarly publication. A sociocognitive approach [2] [3] was adopted to investigate this perplexing issue. The results indicate that participants' citation practices can be better examined as a process influenced by the interplay of cognitive, physical and social factors in accordance with "mind, body and world" [4]. The study further suggests that pedagogy towards citation should move from one-sided rule-governed practice to the integration of social and cognitive dimensions.
\end{abstract}

Keywords-novice Chinese EFL researchers; citation; academic writing; sociocognitive approach

\section{INTRODUCTION}

Written texts occur "at a particular historical moment in a socially specific environment", and is always "oriented toward the 'already uttered' the 'already known' " [5]. Citation, as the bridge across the past and the present, is a common feature of academic writing. However, due to novice EFL researchers' insufficient disciplinary linguistic competence and their unbalanced power relations with potential readers, appropriate citation may be hard to achieve. Reference [6] attributes the inappropriate source use by novice ESL/EFL writers, particularly those from the Far East, to being culturallyconditioned tentatively, representing an essentialized view. Responsive arguments indicate the unreliability of informants and the simplicity of the explanation in this research, and suggest that culture is an important yet not the only influencing factor of cross-cultural source use [7].

Along with the debates on cultural influence on citation, a

*The research is funded by the Research Grant of Department of Education of Shaanxi Province, China (No 16JK1748). growing body of empirical research has examined novice ESL/EFL writers' attitudes towards and perceptions of AngloAmerican literary conventions, such as university students in Japan [8], Korea [9], Latin America [10], Sweden [11], Singapore [12], Vienam [13] and Denmark [14]. In particular, [15] highlights the inherent difficulties in making a determination of plagiarism based on simple text matching between cited words and source texts.

Existing scholarship towards citation practices can actually be quite problematic given the number of factors that guide citation practices of novice writers, particularly without adopting a context-specific approach [15] [16] [17] [18]. Novice writers may carry certain distinctive features. They have limited understandings about when and how to signal the role that a source has played in a new text of their own [1]. For example, they are unable to distinguish forms of plagiarism other than verbatim copying without using quotation marks and/or without giving the source [14]. Reference [19] discusses the difficulties faced by novice academic writers in identifying voices within the sources and developing their own by using the sources. All these are aggregated and escalated when novice researchers write in an EFL context.

This paper explores the possibility of approaching the issue of citation based on naturalistic case study to pursue the underlying infrastructure and discuss why novice writers are employing certain strategies towards their citation practices based on the analyses of their citation types and functions. A sociocognitive approach (henceforth referred to as SCA) is adopted not as the definitive insight into citation practice, but hopefully it can provide a vocabulary for understanding the complex issue and, to some extent, escaping the legal and ethical claims of academic dishonesty and unsuccessful ownership surrounding inappropriate citation.

\section{A SOCIOCOGNITIVE APPROACH TO CITATION PRACTICES}

Learning is the default process of continually aligning oneself with one's sociocognitive environment and more [3] SCA adopts a non-cognitivist view of cognition [4], which deviates from the mainstream of SLA study where cognitive approach takes the lead [20]. As the name itself implies, SCA claims that "individual mind and ecosocial world aren't 
radically separated but rather functionally integrated" [4], and that this functional integration offers a compromise between the cognitive and socio-cultural camps and provides a possible way to understanding SLA.

A sociocognitive approach to language study is indeed embedded in the view that language and its acquisition are not distinctively separated from the rest of the world [3] as they truly are. This approach also marks the major claim that the social (world), the physical (body), and the cognitive (mind), the integrated system, are parts of the concurrent processes underlying the acquisition development. From the SCA perspective, learning and interaction work together in the learning process. Learning is not treated as a rigid way to acquire forms, but as part of people's ability to adapt and survive. Interaction in this sense, rather than viewed as a noncooperative robot-type or a "form of input PRE-processing" [4], proves its fundamental significance in human sociality, including language learning. Learners coordinate or align their activities consciously and continuously for the benefits of their own or the benefits shared with their partners. Correct developmental processes are thus reiterated [21].

In SCA, cognition is seen as "adaptive intelligence" rather than "an isolated computational process" [4]. Accordingly, novice EFL writers' citation practices involve social interaction, or "alignment" in SCA term, as the main driving force. The citing forms and citation manuals composing the major component of traditional teaching are viewed as the input to be processed by the learners in order to align with academic conventions. They are not the ultimate goal of learning, but part of learners' adaptive device. To better pursue novice EFL researchers' citation practices and add empirical evidence to SCA, the current study is guided by the following research questions:

- What are novice Chinese EFL researchers' attitudes, motives and self-initiated strategies to academic citation?

- What are the social and individual factors that underlie their citation practices?

\section{Methodology AND PROCEDURE}

An emic approach elicits information through the informants' eyes, and provides "insider accounts" [22]. Qualitative research approaches that attempt to honor the profound wholeness of social scenes and individual in the world have a central place in the study of the learning and teaching of second/additional languages [2]. Therefore, this study employs introspective methods to obtain information about the inconspicuous mental processes, such as feelings, thoughts, attitudes and motives [23]. Such naturalistic case study is, as [23] concludes, an excellent way to obtain "a thick description" of a complex issue set in a cultural context.

\section{A. Participants}

Considering various types of data to be collected and the ethical sensitivity of citation practices, it would be best to choose participants who are willing to share the details of citing practices as much as possible and available for the time to be devoted. The researcher adopted convenient sampling and invited three novice researchers who were friends to the researcher. Therefore, the conclusion of the current study, to some extent, is contextualized instead of universal. All the three participants, Lucy, Rachel and Calvin (pseudonyms), are enrolled in doctoral programs when data for the research were collected, majoring in social security, public administration, applied chemistry respectively in a major university in East China. They agreed to take part in the study and commented upon one of their academic paper written in English for scholarly publication. They wrote process logs while writing and further explained their citing behavior in discourse-based interviews. Lucy and Rachel are not required for international publication for graduation. Unlike them, Calvin was required to publish at least two articles indexed by SCI (Science Citation Index) to get his degree. All the three participants did not have any overseas study or work experience, and their standardized English test scores are as follows: Lucy (CET 6: 549/710; TOEFL: 91/120), Rachel (CET 6:510/710), Calvin (CET 6: 450/710).

\section{B. Data Collection and Analysis}

Data collection was conducted for seven months with individual differences caused by participants' different writing progress and logistical reasons. A background survey via semistructured interview was conducted for each participant to gather some basic information about their language learning experience, their attitude toward learning a language, their motivation to learn English, their access to academic English training, etc. Process logs, discourse-based interviews, the developing texts and the sources participants used while writing are collected. The background survey and process log template were written in English. Interviews were conducted in Mandarin Chinese, mother tongue for both the participants and the researcher. Their answers were translated into English on the spot and the researcher invited them to see if they agreed with the translation to best take down their thoughts.

The interviews were transcribed and partly translated into English. The interview data as well as the corresponding text, if available, were added to each citation practice. Then the data were analyzed via a recursive process to notice any trends or patterns regarding sets of themes, such as participants' attitudes, motives and self-initiated strategies corresponding to research question one. Individual and societal factors were coded to answer research question two.

\section{FINDINGS}

\section{A. Participants' Attitudes}

Firstly, all the three participants are quite aware of the academic conventions and very cautious about citation practices throughout their writing process. For example, when asked about their view about citation without attributing, Calvin answered decisively: "That is plagiarism. I will definitely not do so."

Lucy and Rachel expressed the same attitude toward verbatim copying and employed different strategies to avoid academic dishonesty. Lucy intentionally searched for APA manual for reference, learned from subject teachers, language courses and published English articles in international journals. 
To avoid verbatim copying, Rachel chose to translate the English sources into Chinese first, and then did the reverse after finishing her Chinese draft. In this way the wording would be certainly different from the source. Rachel commented on the widely used strategy-paraphrasing, and explained her worry about this method:

If I paraphrase from the source, it would bother me a lot since I'm not sure whether the meaning of the original sentence(s) would be distorted by me. So I'm very careful about paraphrasing.

Secondly, participants are very cautious about the accuracy of their citation practices. How to deal with second-hand sources is a very sensitive topic in terms of academic integrity. Rachel pointed out that:

I decided to include the second-hand source in my paper only after I found the article or source and made sure that this source was used correctly in the original paper and relevant to my article.

Lucy noticed that some newly released data mentioned by her supervisor in class could be cited in her article. She said that "My supervisor mentioned this in class. I double-checked it from the government website before I cited it in my paper."

Lucy also showed considerable patience towards the key terms she used in her article. She searched for many articles to decide on the origin of a term-Unified Social Security Model, since she was not sure who was the first scholar proposing such a term. Finally she chose to cite "Yingsheng Li (2007)" as the source of this term for the following reasons. $\mathrm{Li}$, a full professor at a famous university in China, is the first scholar who mentioned the term based on Lucy's search results, and is one of the leading scholars in the field of social security. She thought it would be safe to cite Li's article.

\section{B. Participants' Motives}

Firstly, they have the intention to write in a professional way. Lucy is writing on the Establishment of a Classified Social Assistance System in China, which is of unique Chinese features. Therefore, currently, she only cited articles from Chinese journals. When asked about whether she will cite internationally, say, studies on other countries' welfare systems, she answered: "Yes, I will probably find some articles from international journals. Some review articles would be my best choice. They can be used in my introduction part to make my article sound more professional and international."

Secondly, they want to show their insider status. Calvin listed cited articles after every sentence in his first paragraph in the introduction part.

The first paragraph is to introduce my research background. I read a lot before I start to write. Therefore, you see, I first finish the paragraph, and then find some representative articles to show I'm in this field.

\section{Participants' Self-Initiated Strategies}

Calvin showed apparently different strategic patterns from Lucy and Rachel. He stated that articles published in prestigious journals are his first choices. He said, "I only cite the articles that are from prestigious journals. The quality of journals can be judged by their impact factors, for example." Also, the content is considered more important than publication date. For Calvin, a science student, articles written by other scholars only offer inspiration to his research. He may replicate previous research to test the results, or borrow the experimental methods only, or devise something completely different from the articles he is reading. Novelty and originality is of crucial importance in his discipline. Upon his choosing the citation sources, the sequence of priority is content, practicality, relativity and accuracy. The publication date of the article is of little effect on his choice making.

Things are a little different for Lucy and Rachel. They believed that the latest is the best. Take Rachel's case for an example. She was eager to search for newly published articles and used as her citation in the article although some of them do not offer her any inspiration. For example, when she was writing article, she preferred to use articles published after 2006.

Lucy and Rachel also held the belief that the number and quality of the reference matters. Sources serve different purposes for the three participants. For Lucy and Rachel majoring in social sciences, they thought that citing others' words is an important and inevitable way to get connected to the academia. The number and quality of the reference is an indirect way to show their academic standards. They used a lot citation in their articles, which is a great contrast to Calvin, who only used citations in introduction part with only one or two articles mentioned in the rest.

\section{INFLUENCING SOCIAL AND INDIVIDUAL FACTORS}

\section{A. Social Factors}

As shown in the collected data, a number of social factors contribute to the participants' citing behavior, namely different academic conventions, the influence from their academic supervisors, formal or informal training in academic writing, etc.

The disciplinary differences in citation have been widely discussed by scholars [24]. Practically, differences also exist across cultures. It would be a simplistic view to take cultural differences as a decisive factor accounting for novice researchers' citation practices. It is hard to deny the influence of cultural difference, not only in terms of the way of thinking, but also the corresponding writing conventions. Lucy and Rachel, both as social science students, are much more influenced by their ways of writing academic articles in Chinese. They write their drafts in Chinese and follow the Chinese citing conventions before they work out their English versions. It is partly due to their disciplinary characteristics that their focus is on Chinese society usually and most sources are written in Chinese. It is, therefore, convenient to write a Chinese draft first. Lucy, for example, wrote about Chinese social security system. Scholarship in Chinese would certainly be more authorative and up-to-date. On the other hand, they feel confused about the writing conventions for both Chinese and English academic writing. Comparatively speaking, they have some experiences in Chinese academic writing as they have finished their master's theses and published some articles in Chinese academic journals. To cite Rachel's words, "I 
totally transfer Chinese academic conventions to my English writing. I am not clear about what to cite and how to cite."

Secondly, influence from participants' academic supervisors is also important. Lucy and Rachel clearly mentioned that they used the conventions recommended by supervisors for course papers or even MA thesis. Calvin didn't mention his supervisor's influence in his citing behavior, but did point out that he was advised by his supervisor to write his paper in English, while his first intention was to write a Chinese draft.

Thirdly, formal or informal training in academic writing may have great effects in their citing behavior. All the participants took a course, named Academic English for Communication. Calvin thought he learned a lot from this onesemester course despite of its inappropriate content of the listening part. He learned some academic vocabulary and technical writing skills. On the contrary, Lucy and Rachel gained little from the course. They thought the content is more favorable for science students. As for the informal training, mostly coming from their peers who had some experience in English publication, Calvin mentioned several times about his consultation from senior students in the same program. In contrast, Lucy and Rachel seem to get limited help from peers.

\section{B. Individual Factors}

Firstly, novice writers are not disoriented in citation practices as stated in a lot of scholarship [25]. They are quite conscious of their citation practices and may use them strategically. In order to appear to be catching up with the latest development in her research field, Rachel has exchanged some "old" sources with the latest ones, even though the new ones are not directly related to their research to some extent. She intentionally cited articles published after 2006. For those old ones published before 2000 , even if they are very informative, she is very cautious about listing them in her references. She explicitly pointed out her disposing of one source published in 1981 and exchanged with a more recent article despite that she had to sacrifice the accuracy of the sentence to some extent. Also, some ideas derived from her Chinese reading were also followed by some English sources to prove to readers her wide access to sources. Similar points were stated in [26], which included interview data of criminologists' views of citation, some informants reiterate the importance of recent literature in helping persuade the readership:

"...if you're trying to show that you are writing something that's credible and authorative...including new references suggests to the reader you're keeping yourself on top of your field." (p.482)

Further, Calvin mentioned his reluctance to employ any negative words (e.g. fail, overlook, exaggerate or ignore) to describe other researchers' work. It is proved by [24] which shows that only the humanities and social sciences articles in his corpus containing reporting verbs signifying the unreliability of the source. Instead, Calvin, as a science scholar, recommended a mitigative strategy could be used to deal with the case, that is, either ignore the source or state in a vague way, such as "some studies" without mentioning the author of the source.
Secondly, citation practices are also affected by participants' personal experiences. Calvin is the most confident writer of the three, which may be related to his disciplinary feature and his academic training experience. As a student majoring in applied chemistry, Calvin is surrounded by experienced writers. He has mentioned that he can always get help from his peers in terms of writing conventions for many of his peers have published internationally. In the background survey, he said that he has been receiving training in writing academic papers since undergraduate program, which definitely contributes to his academic confidence and experience. In contrast, Lucy and Rachel didn't receive any training in academic writing before their master's programs, and at the same time few peers of them get published in international journals. Further, when they have difficulty in writing English papers, writing convention manuals or articles in international journals are their favorite choice.

Thirdly, at times novice writers, novice L2 writers in particular, are not skillful at activating and bringing up the most salient information to the needed attention level by the readers. Rachel's writing experience serves as a good example. One reviewer of her article was confused about the background of the research since he/she believes that Chinese are not allowed to have a second child based on the one-child policy, and the fact is that a second child is permitted if the families live in rural areas or each of the couple is the only child in his/her respective family. As cited below:

Reviewer: The stated purpose is to examine how migration influences fertility. However, fertility in China is restricted by the one-child policy (which results in a deviation from the natural sex ratio at birth)

This misunderstanding is caused by the writer's failure to share needed information with the reader or in other words by the gap between the common ground and actual communicative communication.

\section{CONCLUSION AND LIMITATIONS}

Writing academic papers is just like conducting a dialogue between scholars. The differences lie in writers' ignorance and unpredictability of the target readers. Both parties are confined by their social and individual traits, which may bring complexity to the issue. Novice EFL researchers' strategies towards academic citation are devised and adjusted to meet their academic environment and the requirements by international scholarly publication as so to obtain the benefits and survive. Lucy and Rachel initiating to write their first drafts in Chinese is just a case in point. They start from some point approachable and then make adjustment continuously. The collected data and analysis afterwards clearly show that citing forms and citation manuals are important for novice researchers' success, but contribute only in a rather limited way. A myriad of factors may influence the citation practices of this group. Their starting point is not the prescribed conventions, which are just one building block in the whole process. Their practices are largely guided and influenced by the complex social and individual factors and also the interweaving of the two. 
It should also be noted that, just like any other learning process other than language learning, the efforts made by the novice EFL researchers may turn out to be bad. When they underestimate the rigidity of the citation conventions in AngloAmerican context, they may be accused of plagiarism. When they stick to the conventions strictly at times, causing overcitation for example, they may reveal their novelty at first sight

Each study has its limitations, and the current study is no exception. The analysis of the study is based on convenient sampling and its size is small. The conclusion may not be comprehensive and universally-based. Besides, the data of their citation practices were collected from one of their academic writing respectively, which may lead to contextualized conclusion although that accords with the basic rationale of this study, that is, citation practice, as an integral and important component of academic writing, is situated and mediated by institutional discourses and practices.

\section{REFERENCES}

[1] D. Pecorari, and P. Shaw, "Types of student intertextuality and faculty attitudes," Journal of Second Language Writing, vol. 21, pp. 149-164, 2012.

[2] D. Atkinson, "Toward a sociocognitive approach to second Language acquisition," The Modern Language Journal, vol. 86, pp. 525-545, 2002.

[3] D. Atkinson, E. Churchill, T. Nishino and H. Okada, "Alignment and interaction in a sociocognitive approach to second language acquisition," The Modern Language Journal, vol. 91, pp. 169-188, 2007.

[4] D. Atkinson, "Language learning in mindbodyworld: A sociocognitive approach to second language acquisition, " Language Teaching, vol. 47, pp. 467-483, 2013.

[5] M. Bakhtin, "Discourse in the novel," in The Dialogic Imagination: Four Essays, M. Holquist and C. Emerson, Trans. Austin: University of Texas, 1981, pp. 259-422.

[6] C. Sowden, "Plagiarism and the culture of multilingual students in higher education abroad, " ELT Journal, vol. 59, pp. 226-233, 2005.

[7] D. Liu, "Plagiarism in ESOL students: Is cultural conditioning truly the major culprit," ELT Journal, vol. 59, pp. 234-241, 2005.

[8] G. Wheeler, "Plagiarism in the Japanese universities: Truly a cultural matter?," Journal of Second Language Writing, vol. 18, pp. 17-29, 2009.

[9] E. H. Rhee, "Complexities and dynamics of Korean graduate students' textual borrowing in academic writing," TEMPLE UNIVERSITY, unpublished doctoral dissertation, 2010.

[10] Vasconcelos, S., Leta, J., Costa, L., Pinto, A., and Sorenson, M. M. (2009) Discussing plagiarism in Latin American science. EMBO reports 10:677-682.

[11] D. Razera, H. Verhagen, T. C. Pargman, and R. Ramberg, (2010, June) "Plagiarism awareness, perception, and attitudes among students and teachers in Swedish higher education-a case study," 4th International Plagiarism Conference-Towards an authentic future, Northumbria University in Newcastle Upon Tyne-UK, pp. 21-23, June 2010.

[12] A. Chandrasegaran, "Cultures in contact in academic writing: Students' perceptions of plagiarism," Asian Journal of English Language Teaching, vol. 10, pp. $91-113,2000$.

[13] L. H. Phan, "Plagiarism and overseas students: stereotypes again?" ELT Journal, vol. 60, pp. 76-78, 2006.

[14] I. Klitgård, "From copy-and-paste to trace-and-learn: A qualitative survey of student perceptions of plagiarism", Zeitschrift Schreiben, pp. 1-7. Retrieved from: http://www.zeitschriftschreiben.eu/Beitraege/klitgard_Qualitative_Survey.pdf, 2009.
[15] E. J. Eckel, “Textual appropriation in engineering Master's theses: A preliminary study," Science and Engineering Ethics, vol. 17, pp. 469483, 2011.

[16] R. Chandrasoma, C. Thompson, and A. Pennycook, "Beyond plagiarism: Transgressive and nontransgressive intertextuality," Journal of Language Identity, and Education, vol. 33, pp. 171-193, 2004.

[17] D. Pecorari, "Good and original: Plagiarism and patchwriting in academic second-language writing," Journal of Second Language Writing, vol. 12, pp. 317-345, 2003.

[18] L. Shi, "Textual appropriation and citing behaviors of university undergraduates," Applied Linguistics, vol. 31, pp. 1-24, 2010.

[19] S. Angélil-Carter, Stolen Language?: Plagiarism in Writing, 2nd ed. Harlow: Longman, 2000.

[20] S. M. Gass, J. Lee, and R. Roots, "Firth and Wagner (1997): New ideas or a new articulation?" The Modern Language Journal, vol. 91, pp. $788-799,2007$.

[21] K. Hill, “Comments on J. Zuengler and E. R. Miller's 'cognitive and sociocultural perspectives: Two parallel SLA worlds?"' TESOL Quarterly, vol. 40, pp. 819-826, 2006.

[22] N. Harwood, "An interview-based study of the functions of citations in academic writing across two disciplines," Journal of Pragmatics, vol. 441, pp. 497-518, 2009.

[23] Z. Dörnyei, Research Methods in Applied Linguistics: Quantitative, Qualitative, and Mixed Methodologies, Oxford: Oxford University Press, 2007.

[24] K. Hyland, "Academic attribution: Citation and the construction of disciplinary knowledge," Applied Linguistics, vol. 20, pp. 341-367, 1999.

[25] K. Mansourizadeh, and U. K. Ahmad, "Citation practices among nonnative expert and novice scientific writers," Journal of English for Academic Purposes, vol. 10, pp. 152-161, 2011.

[26] P. Rock, "Chronocentrism and British criminology," The British journal of Sociology, vol. 56, pp. 473-491, 2005. 